Louisiana State University

LSU Digital Commons

$5-16-2011$

\title{
Creation of 3D digital anthropomorphic phantoms which model actual patient non-rigid body motion as determined from MRI and position tracking studies of volunteers
}

C. M. Connolly

University of Massachusetts Medical School

A. Konik

University of Massachusetts Medical School

P. K.R. Dasari

University of Massachusetts Medical School

P. Segars

Duke University

S. Zheng

University of Massachusetts Medical School

See next page for additional authors

Follow this and additional works at: https://digitalcommons.Isu.edu/physics_astronomy_pubs

\section{Recommended Citation}

Connolly, C., Konik, A., Dasari, P., Segars, P., Zheng, S., Johnson, K., Dey, J., \& King, M. (2011). Creation of 3D digital anthropomorphic phantoms which model actual patient non-rigid body motion as determined from MRI and position tracking studies of volunteers. Progress in Biomedical Optics and Imaging Proceedings of SPIE, $7964 \mathrm{https://doi.org/10.1117/12.878193}$

This Conference Proceeding is brought to you for free and open access by the Department of Physics \& Astronomy at LSU Digital Commons. It has been accepted for inclusion in Faculty Publications by an authorized administrator of LSU Digital Commons. For more information, please contact ir@lsu.edu. 


\section{Authors}

C. M. Connolly, A. Konik, P. K.R. Dasari, P. Segars, S. Zheng, K. L. Johnson, J. Dey, and M. A. King 
University of Massachusetts Medical School

eScholarship@UMMS

2011-05-02

\section{Creation of 3D Digital Anthropomorphic Phantoms which Model Actual Patient Non-rigid Body Motion as Determined from MRI and Position Tracking Studies of Volunteers}

Caitlin M. Connolly

University of Massachusetts Medical School

Et al.

\section{Let us know how access to this document benefits you.}

Follow this and additional works at: https://escholarship.umassmed.edu/ssp

Part of the Life Sciences Commons, and the Radiology Commons

\section{Repository Citation}

Connolly CM, Konik A, Dasari PK, Segars P, Zheng S, Johnson KL, Dey J, King MA. (2011). Creation of 3D Digital Anthropomorphic Phantoms which Model Actual Patient Non-rigid Body Motion as Determined from MRI and Position Tracking Studies of Volunteers. Senior Scholars Program. https://doi.org/ 10.13028/0zxz-e851. Retrieved from https://escholarship.umassmed.edu/ssp/119

This material is brought to you by eScholarship@UMMS. It has been accepted for inclusion in Senior Scholars Program by an authorized administrator of eScholarship@UMMS. For more information, please contact Lisa.Palmer@umassmed.edu. 
Creation of 3D digital anthropomorphic phantoms which model actual patient non-rigid body motion as determined from MRI and position-tracking studies of volunteers

C.M. Connolly ${ }^{1}$, A. Konik' ${ }^{1}$, P.K.R. Dasari ${ }^{1}$, P. Segars ${ }^{2}$, S. Zheng ${ }^{1}$, K.L. Johnson ${ }^{1}$, J. Dey ${ }^{1}$, M.A. King ${ }^{1}$

${ }^{1}$ University of Massachusetts Medical School, Worcester, MA, USA ${ }^{2}$ Duke University Medical School, Durham, NC, USA

\section{INTRODUCTION}

The purpose of this study is to create $3 \mathrm{D}$ digith anthroponorphic phantoms based on MRI data of volunteers undergoing a series of clinically relevant motions. These phantoms with combined position tracking will be used to investigate both imaging-data-driven and motion-tracking strategies to estimate and correct for patient motion. Our initial application will be to cardiac-perfusion SPECT imaging where the voxelized XCAT phantoms will model patient activity and attenuation distributions for each volunteer with corresponding motion-tracking data from the markers on the body-surface. Monte Carlo methods will then correction strategies.

\section{METHOD}

MRI Acquisition MRI of the volunteers was performed with a 3-T whole body scanner (Achieva, Philips Medical System) using the built-in quadrature body coil. All scans were performed without the use of contrast agents. During the imaging session, volunteers were in the supine position with arms over their head as in clinical SPECT cardiac-perfusion imaging. The MR images of the torso on each volunteer were acquired in sagittal orientation with single shot real-time EKG and Navigator gated sequence (2D T1-Fast Field Echo, $\mathrm{TR} / \mathrm{TE}=5.5 / 3.1$, slices $=117$, acquisition matrix $=128 \times 100$, voxel size $=3 \mathrm{~mm}$ ). To obtain a significant contrast between the blood pool and heart tissue, an appropriate trigger delay was calculated based on the subject's heart rate, which delays the sequence at the steady diastolic stage of the cardiac cycle. Transverse

MRI of a human volunteer, obtained with an EKG triggered
Navigator sequence. The blood Navigator sequence. The blood pool and myocardium of the lea
ventricle are clearly visible, as shown at right. Transverse and coronal slices were reforms
from the sagittal acquisitions.
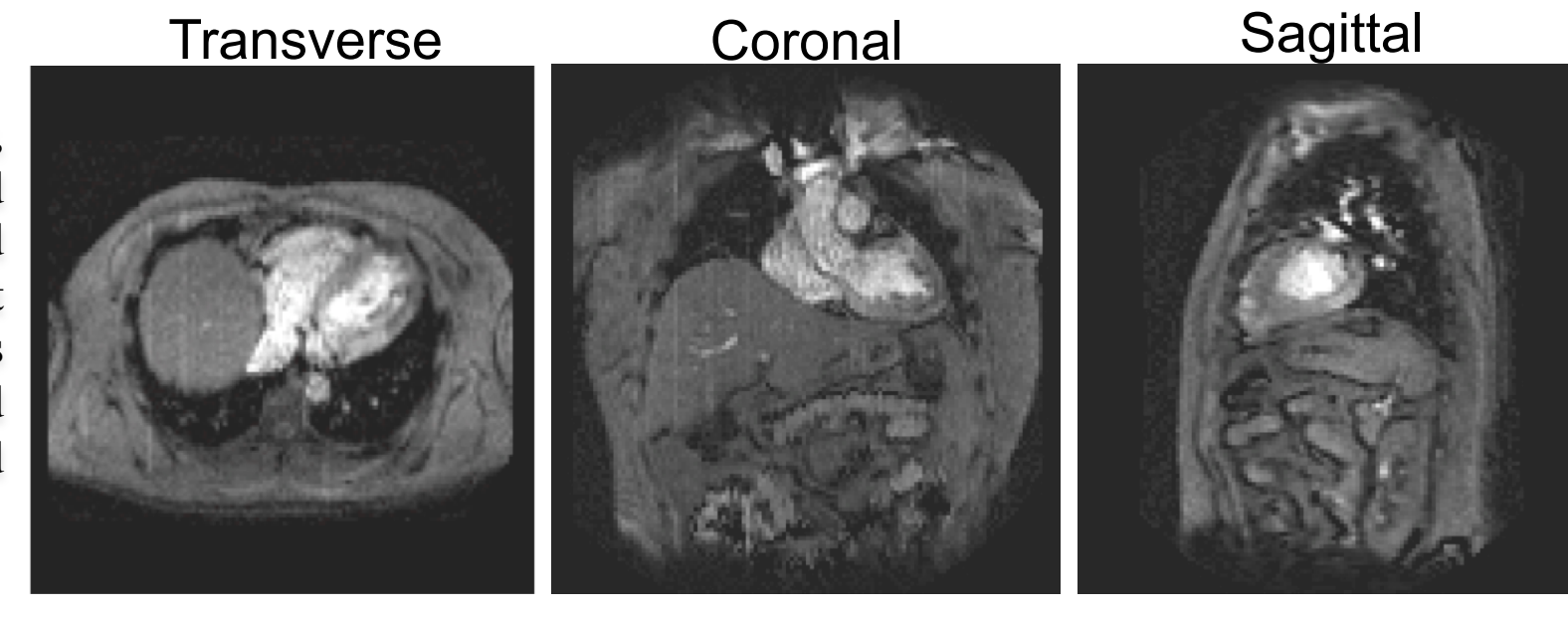

To capture motion states, a modified Navigator sequence consisting of 59 EKG triggered sagittal slices covering the entire thorax with $3 \mathrm{~mm}$ gaps between the $3 \mathrm{~mm}$ thick slices was obtained. These sequences were captured at end-expiration using MotionTrak Display window (shown below), which is a time sequence of $1 \mathrm{D}$ plots across the diaphragm. This allowed the researcher to provide feedback to volunteer to after each inhale return their end-expiration position to accepted level for MRI acquisition. Shown at right is the MotionTrak display window. Red dots mark lines outline the respiratory gate window, and the green lines show

navigator beam height. Green dashes at the bottom indicate

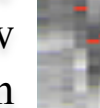

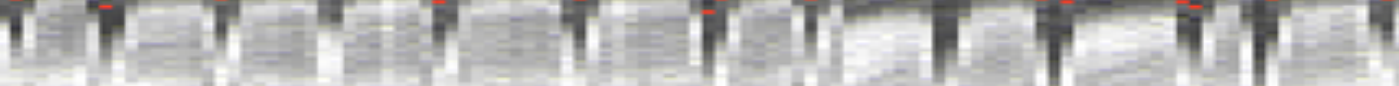

External Motion Tracking During MRI, volunteer motion was tracked using the Vicon visual tracking (V) , a stereo tracking system, consisting of 3 near-infra-red cameras, as shown below. The motion information obtained through the tracking of the reflective markers on the chest and abdomen of the volunteers is temporally correlated to the motion of the structures internally as seen in MRI.

In the figure on the left are VTS cameras used for tracking the the calibration phantom for coordinate systems. On the right is the volunteer with
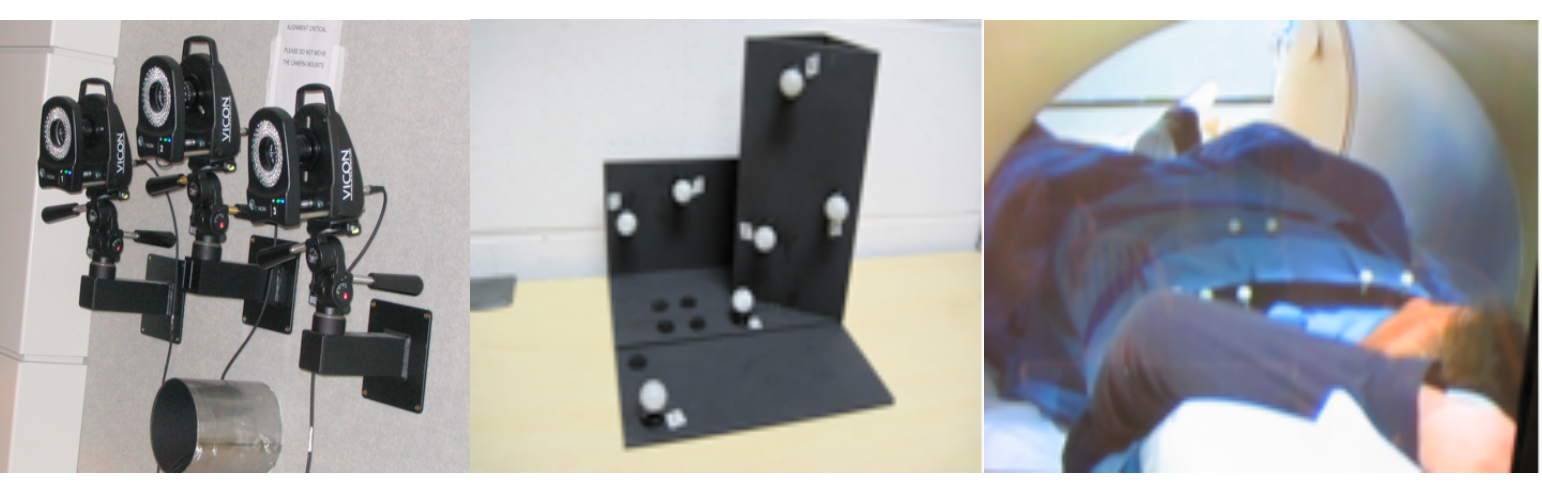

XCAT phantom creation We created individual-specific extended cardiac-torso (XCAT) phantoms [1] fit to our volunteer MRI imaging data using an interactive graphical user interface (GUI) (shown below) developed by Dr. Segars, which allows a user to adapt the NURBS-based structures of the XCAT phantom to tomographic data. We used the Navigator sequence covering the complete torso to match the XCAT phantom to the volunteer's specific anatomy based on MRI. This phantom represented the pre-motion state. The volunteer specific phantom was then fitted to the post-motion navigator MRI, creating the poststate. The volunteer spe
motion XCAT phantom.

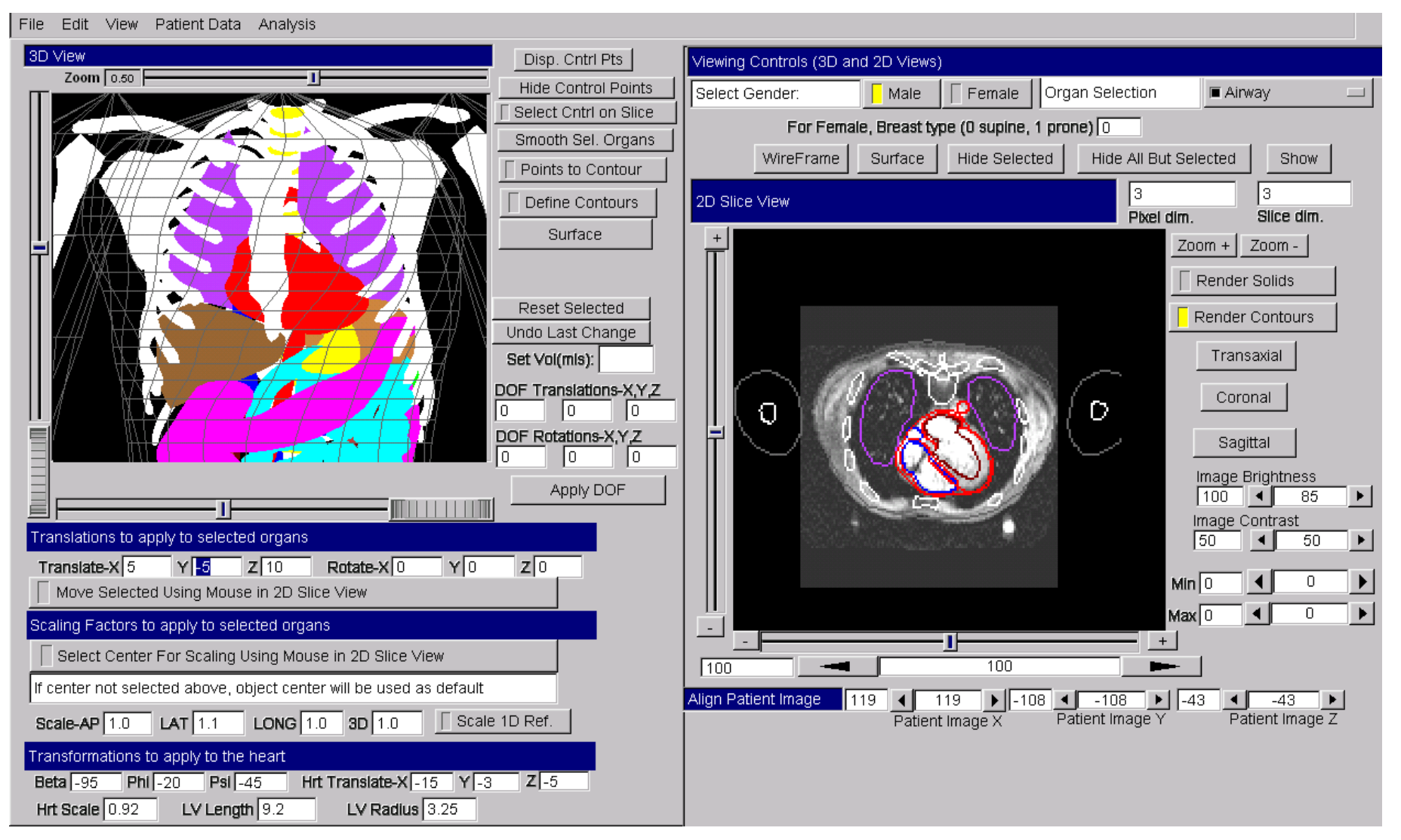

RESULTS

Examples of multiple motion states including an axial slide (on the left), which represents a 6-DOF rigid motion, and a shoulder twist (on the right), a
non-rigid body movement, are shown below. The XCAT phantoms overlaid on MRI images are shown for the motions in rows A and B (stripping in non-rigid body movement, are shown below. The XCAT phantoms overlaid on MRI images are shown for the motions in rows A and B (stripping in
MRI due to gaps between sagittal slices), while the voxelized XCAT phantoms representing overlaid emission and attenuation distributions are shown MRI due to gaps between sagittal slices), while the voxelized XCAT phantoms representing overlaid emission and attenuation distributions are shown respective
Rigid Motion - Axial Slide
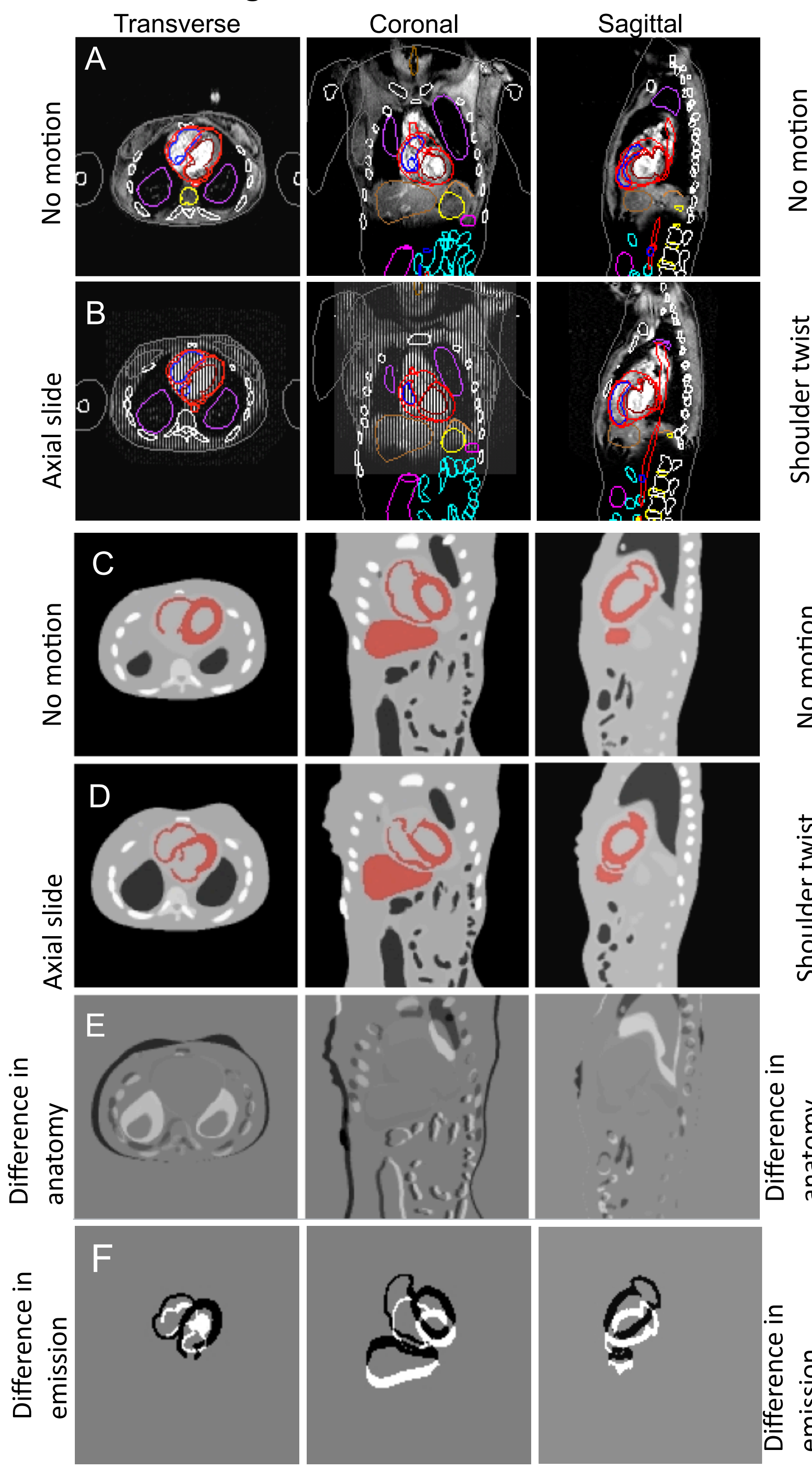

Non-rigid Motion - Shoulder Twist
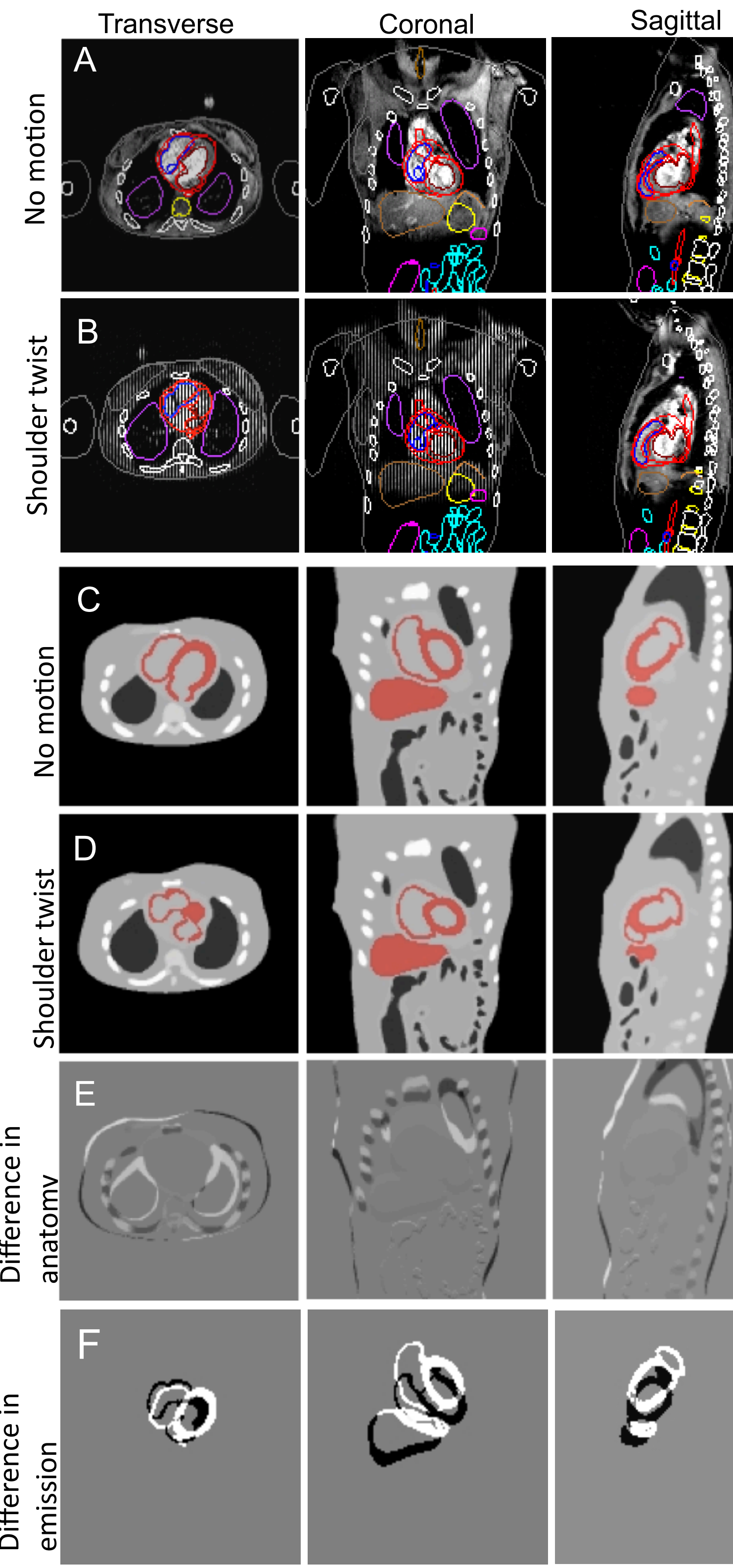

We successfully segmented the organs from the MRI data to create 3D voxelized phantoms representing different motion states, including both rigid and non-rigid body motion. The internal organ motion data obtained from MRI, will be correlated with external motion tracking data for developing and evaluating motion correction methods. We plan to use the phantoms initially for performing cardiac SPECT simulations to improve motion correction algorithms.

\section{ACKNOWLEDGEMENT}

This work was supported by the National Institute of Bio-medical Imaging and Bioengineering NIBIB Grant No. R01 EB001457 and by Philip Medical Systems. The contents are solely the responsibility of the authors and do not necessarily represent the official views of the National Institutes of Health or Philips Medical Systems.

REFERENCES

[1] W. P. Segars, M. Mahesh, T. J. Beck, E. C. Frey, and B. M. Tsui, "Realistic CT simulation using the 4D XCAT phantom," Med Phys, vol. 35, pp. 3800-8, (2008). 